\title{
Mu Glutathione-S-Transferase to Creatinine Ratio Measurement
}

National Cancer Institute

\section{Source}

National Cancer Institute. Mu Glutathione-S-Transferase to Creatinine Ratio

Measurement. NCI Thesaurus. Code C79458.

The determination of the ratio of mu glutathione-s-transferase compared to creatinine present in a sample. The measurement may be expressed as a ratio or percentage. 